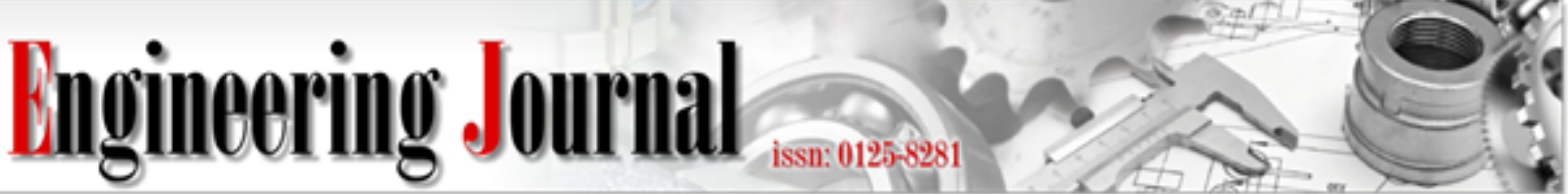

Article

\section{Hierarchical Decentralized LQR Control for Formation-Keeping of Cooperative Mobile Robots in Material Transport Tasks}

\author{
Hendi Wicaksono Agunga and Itthisek Nilkhamhangb,* \\ School of Information, Computer, and Communication Technology, Sirindhorn International \\ Institute of Technology, Thammasat University, Thailand \\ E-mail: ahendi@staff.ubaya.ac.id, b,*itthisek@siit.tu.ac.th (Corresponding author)
}

\begin{abstract}
This study provides a formation-keeping method based on consensus for mobile robots used in cooperative transport applications that prevents accidental damage to the objects being carried. The algorithm can be used to move both rigid and elastic materials, where the desired formation geometry is predefined. The cooperative mobile robots must maintain formation even when encountering unknown obstacles, which are detected using each robot's on-board sensors. Local actions would then be taken by the robot to avoid collision. However, the obstacles may not be detected by other robots in the formation due to line-of-sight or range limitations. Without sufficient communication or coordination between robots, local collision avoidance protocols may lead to the loss of formation geometry. This problem is most notable when the object being transported is deformable, which reduces the physical force interaction between robots when compared to rigid materials. Thus, a decentralized, hierarchical LQR control scheme is proposed that guarantees formation-keeping despite local collision avoidance actions, for both rigid and elastic objects. Representing the cooperative robot formation using multiagent system framework, graph Laplacian potential and Lyapunov stability analysis are used to guarantee tracking performance and consensus. The effectiveness and scalability of the proposed method are illustrated by computer simulations of line ( 2 robots) and quadrilateral (4 robots) formations. Different communication topologies are evaluated and provide insights into the minimum bandwidth required to maintain formation consensus.
\end{abstract}

Keywords: Formation-keeping, cooperative mobile robots, hierarchical decentralized LQR control, material transport, multi-agent system, formation consensus.

ENGINEERING JOURNAL Volume 25 Issue 12

Received 23 April 2021

Accepted 19 December 2021

Published 30 December 2021

Online at https://engj.org/

DOI:10.4186/ej.2021.25.12.37 


\section{Introduction}

Mobile robots are often used in industrial applications to transport materials and inventories in factories, warehouses [1], and assembly lines [2]. A challenging problem involves objects that may be oversized and/or overweight, potentially exceeding the capacity of a single transport robot [3, 4]. Examples include large shelves [5], automobiles [6], and cargo containers [7]. Instead, this task can be achieved by multiple robots working together [8]. However, this cooperative, multi-robot system requires advanced formation control strategies to avoid accidental damage to the transport object and the robots [9].

There are several ways in which material transport can be accomplished through the cooperation of multiple robots. Elio Tuci et al. reviewed different material transport strategies based on pushing, caging, and grasping [8]. Pushing strategies consist of pushing an object that is not physically attached to the robots [10]. Similarly, caging strategies involve the robots entrapping the object and maintaining firm pressure during transport [11]. Alternatively, in grasping strategies, the robots physically attach to the object by some mechanism, such as grippers or suctions, and transport is conducted by pushing, pulling, or both. This prevents the object from falling during transportation. Certain systems also allow the robots to lift the object [12]. In the survey [8], an object can also be simultaneously placed on top of multiple robots for transport, which is categorized as a type of grasping strategy since the robot forces are aligned, and physical contact with the object is maintained throughout the transportation process. For omni-directional robots, a flexible mechanism or supporting joint should be carefully designed so as not to interfere with the robots' omni-directional capabilities. Some examples include mechanical grippers [13], a rotary platform placed on top of the robot's base [14], and joints with displacement-based force sensors [15]. Examples of these cooperative transport systems in which the object is placed on top of the robots can be found in $[16,17,18]$, and serve as the motivation for this work.

An important part of cooperative material transport strategies is formation-keeping, which ensures that the object does not fall or be otherwise damaged [19]. To achieve formation control, multiple robots form and maintain geometric shapes using a consensus scheme [20]. The two main objectives in formation control are aggregation and trajectory tracking [21]. Aggregation involves the convergence of all agents from separate locations to a predefined geometric shape, such as a line, wedge, or quadrilateral formation. After this process is completed, all robots in the group would move in unison according to a certain trajectory while maintaining formation. In some scenarios, the formation may temporarily change depending on environmental requirements, such as when encountering obstacles or passing through a narrow doorway [22]. Examples of multirobot applications that allow for changes to the formation are search-and-rescue, patrols, and robot flocking [23]. However, this is not applicable to industrial material transport systems, where significant changes to the formation geometry that may occur due to collision avoidance actions or other disturbances can cause the object to fall or be damaged. This remains a major problem in formation control [24], especially if the formation cannot deviate from the predefined patterns.

To address this issue, a number of algorithms have been proposed to maintain formation during transport, including virtual structure and leader-follower. In the virtual structure method, the robot formation is considered a single rigid structure [25], typically implemented as a centralized scheme, which is susceptible to singlepoint failures that can disrupt the entire formation [26]. Leader-follower control was proposed to solve this problem, in which information is assumed to be fully available to the lead robot, such as the desired trajectory and external operation commands, that are then distributed to the followers [27]. The leader-follower control architecture could achieve consensus for formation-keeping, even with no direct communication channels between the robots, due to physical force interactions that act as an indirect form of information exchange [28]. However, the leader-follower strategy is a semi-centralized method and not robust since it is dependent on the leader [29]. If the lead robot malfunctions or communication failure occurs, then the follower robots cannot continue to operate [30].

This research is motivated by using the physical force interactions between robots as an indirect communication method. However, if the material being transported is soft or flexible, the robot formation may become significantly deformed. This problem is most notable when local detection of an unknown obstacle occurs and collision avoidance protocols are taken by a robot in the formation. The obstacle may not be detected by other robots in the group due to line-of-sight or range limitations. This causes disagreement that negatively affects the formation geometry. In severe cases, the object being transported may fall or become damaged. To avoid this, hierarchical, decentralized LQR control is proposed that uses local communication between robots to implement a virtual spring structure that is capable of formation-keeping, regardless of the rigidity of the transport material. In this study, undirected graph theory is used to describe physical force interaction and communication exchange between robots. An LQR-based controller is designed that guarantees the sta- 
bility of trajectory tracking errors. A consensus controller is then constructed using information exchange between agents to achieve convergence to the average consensus state of the group. Each robot implements a local collision avoidance protocol based on an artificial potential function upon detecting unexpected obstacles. The proposed method is evaluated using computer simulations of line (two robots) and quadrilateral (four robots) formations. The effectiveness is shown for both rigid and elastic materials, with notable improvement in formation-keeping performance over conventional, decentralized LQR control. The contribution of the consensus gain in the hierarchical LQR controller is demonstrated, with higher gains ensuring faster consensus convergence but requiring more control effort. Lastly, the effect of communication topology is examined and provides insights into the minimum bandwidth required to successfully implement this strategy.

\section{Background and Preliminaries}

\subsection{Graph Theory Notations}

In a multi-agent system (MAS), graph theory is a well-known mathematical tool for interaction analysis between agents. The communication network topology expressed by a graph can be categorized as undirected or directed. A directed graph is a set of nodes connected by edges, with each edge having a direction associated with it. Meanwhile, in an undirected graph the edges are bidirectional. Hence, the graph can be traversed in both directions [31]. In this research, the physical interaction between robots are modeled as a mass-spring system that can be represented using an undirected graph, where each robot is a node and the springs act as bidirectional edges.

An undirected graph is defined as $\mathcal{G}=(\mathcal{V}, \mathcal{E}, \Gamma)$ where $\mathcal{V}=\left\{\mathcal{V}_{1}, \mathcal{V}_{2}, \ldots, \mathcal{V}_{N}\right\}$ is a finite, non-empty node set with $N \geq 2, \mathcal{E} \subseteq \mathcal{V} \times \mathcal{V}$ is a set of edges connecting the nodes, $\mathcal{V}_{i}=\{j \in \mathcal{V}:(j, i) \in \mathcal{E}\}$ represents the $i$-th node neighbors. The adjacency matrix $\Gamma \in \mathbb{R}^{N \times N}$ of the graph $\mathcal{G}$ is defined such that $\gamma_{i j}=1$ if $(j, i) \in \mathcal{E}$, while $\gamma_{i j}=0$ if $(j, i) \notin \mathcal{E}$. The in-degree of node $i$ is $D_{i j}=\sum_{j=1}^{N} \gamma_{i j}$ and leads to the definition of the in-degree matrix $\mathbf{D}=\operatorname{diag}\left\{D_{i j}\right\} \in \mathbb{R}^{N \times N}$. Next, $\mathcal{L}=\mathbf{D}-\boldsymbol{\Gamma}$, where $\mathcal{L}$ is known as the Laplacian matrix that describes the connectivity of the topology. The Laplacian matrix $\mathcal{L} \in \mathbb{R}^{N \times N}$ of the graph $\mathcal{G}$ is defined as $l_{i i}=\sum_{j \neq i} \gamma_{i j}$ and $l_{i j}=-\gamma_{i j}$ for all $i \neq j$ [32]. An important feature of $\mathcal{L}$ is that the sums of all rows in $\mathcal{L}$ are zero, thus $\mathbf{1}_{N}=[1,1, \ldots, 1]^{T} \in \mathbb{R}^{N}$ is an eigenvector of $\mathcal{L}$ associated with the eigenvalue $\lambda=0$; then, $\mathcal{L} 1_{N}=0_{N}$ [33]. The subsequent graph theory properties are relevant to this paper.
Lemma 1. [32, 33] An undirected graph is called connected if and only if the Laplacian matrix $\mathcal{L}$ has exactly one zero eigenvalue and all of the non-zero eigenvalues are positive, with $\operatorname{rank}(\mathcal{L})=N-1$.

Lemma 2. [34] For an undirected graph, $\gamma_{i j}=\gamma_{j i}$ and $\boldsymbol{\Gamma}=\boldsymbol{\Gamma}^{T} \in \mathbb{R}^{n \times n}$. Then, $\operatorname{null}(\boldsymbol{\Gamma})=\left\{\boldsymbol{x} \in \mathbb{R}^{n} \mid x^{T} \boldsymbol{\Gamma} \boldsymbol{x}=\right.$ $0\}$ if and only if $\boldsymbol{\Gamma}$ is positive semi-definite or negative semidefinite, i.e., $\boldsymbol{\Gamma} \geq 0$ or $\boldsymbol{\Gamma} \leq 0$.

\subsection{Graph Laplacian Potential}

This section describes the properties of the graph Laplacian potential that was introduced in [32] for an undirected topology with 0 and 1 adjacency elements.

Lemma 3. $[33,35,36]$ Let $x=\left[x_{1}, x_{2}, \ldots, x_{N}\right]^{T}$; then, the Laplacian potential for an undirected graph described by the Laplacian matrix $\mathcal{L}$ is defined as

$$
V_{L}=\frac{1}{2} \sum_{i, j=1}^{N} \gamma_{i j}\left(x_{i}-x_{j}\right)^{2}=x^{T} \mathcal{L} x
$$

where $\gamma_{i j}=\gamma_{j i}$ (from Lemma 2).

The Laplacian potential function is closely associated with the consensus of the MAS, as is described in Lemma 4.

Lemma 4. [33, 37] For a connected, undirected graph, nodes $(i, j)$ are in agreement if and only if $x_{i}=x_{j}$. This implies that consensus is achieved when $V_{L}=0$.

An undirected graph has obvious physical interpretations. For instance, a group of agents connected by springs, where $\gamma_{i j}$ represents the spring constant between nodes $i$ and $j$, is an undirected graph. The potential energy stored in the springs can then be expressed as the graph Laplacian potential (Eq. 1).

\section{System Description}

The cooperative robot formation is formulated using a mass-spring model, where the agents are expressed as mass nodes connected by spring elements representing physical force interactions through the transport material.

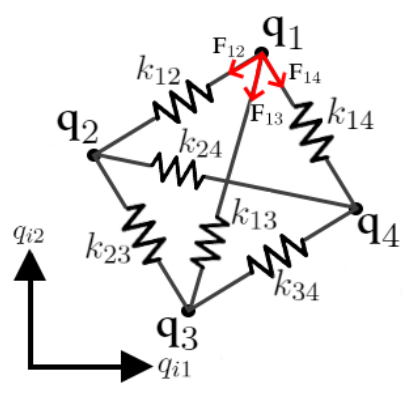

Fig. 1. A quadrilateral robot formation expressed as a mass-spring model. 


\subsection{Mass-Spring Formation Model}

Assume a two-dimensional formation with $N$ robots, where $N \geq 2$. The robots are connected by linear spring elements with no damping and each have a mass $m_{i}>0$, where $i=\{1, \ldots, N\}$. This mass-spring formation model is an undirected graph, and is shown in Fig. 1 for four nodes, labeled as $\mathbf{q}_{1}, \mathbf{q}_{2}, \mathbf{q}_{3}$, and $\mathbf{q}_{4}$. Each node is associated with a position expressed in the Cartesian coordinates of the world frame, $\mathbf{q}_{i}=\left[q_{i 1}, q_{i 2}\right]^{T}$. The spring constant between robots $\mathbf{q}_{i}$ and $\mathbf{q}_{j}$ is represented by $k_{i j}>0$.

When the formation is in its equilibrium state, the springs are at rest with a length defined as $r_{i j}$. Let the relative distance $d_{i j}$ between robots be given by $d_{i j}=\left\|\mathbf{q}_{i}-\mathbf{q}_{j}\right\|$. The forces exerted by all connected springs are obtained from Hooke's law and can be shown as

$$
\mathbf{F}_{i j}=k_{i j}\left(1-\frac{r_{i j}}{d_{i j}}\right)\left(\mathbf{q}_{i}-\mathbf{q}_{j}\right)
$$

where $\mathbf{F}_{i j} \in \mathbb{R}^{2}$ is the interaction force between $\mathbf{q}_{i}$ and $\mathbf{q}_{j}$ through the spring $k_{i j}$. Note that at the equilibrium state, $d_{i j}=r_{i j}$ and the spring contributes no physical force to either robot.

The dynamical equation of motion for robot $\mathbf{q}_{i}$ can be derived as

$$
m_{i} \ddot{\mathbf{q}}_{i}+\sum_{j \in \mathcal{V}_{i}} \mathbf{F}_{i j}\left(\mathbf{q}_{i}, \mathbf{q}_{j}\right)=\mathbf{F}_{i}
$$

where $\mathbf{F}_{i} \in \mathbb{R}^{2}$ is the input force to the robot.

Assuming that all nodes have equal mass (i.e. $m_{i}=$ $m, \forall i)$, and are fully-connected with uniform stiffness (i.e. $k_{i j}=k, \forall(i, j)$ ), then the linearized equation of motion for the mass-spring formation becomes

$$
\mathbf{M} \ddot{\mathbf{q}}+\mathbf{K}_{s} \mathbf{q}=\mathbf{F}
$$

where $\mathbf{M}=\left(m \mathbf{I}_{2}\right) \otimes \mathbf{I}_{N} \in \mathbb{R}^{2 N \times 2 N}, \mathbf{F} \in \mathbb{R}^{2 N}$, $\mathbf{q}=\left[\begin{array}{lll}\mathbf{q}_{1}^{T} & \ldots & \mathbf{q}_{N}^{T}\end{array}\right]^{T} \in \mathbb{R}^{2 N}$, and $\otimes$ is the Kronecker product. The stiffness matrix $\mathbf{K}_{s} \in \mathbb{R}^{2 N \times 2 N}$ is obtained by linearizing Eq. 2 around the equilibrium state as

$$
\mathbf{K}_{s}=\left[\begin{array}{cccc}
(N-1) \mathbf{K} & -\mathbf{K} & \ldots & -\mathbf{K} \\
-\mathbf{K} & (N-1) \mathbf{K} & \ldots & -\mathbf{K} \\
\vdots & \vdots & \ddots & \vdots \\
-\mathbf{K} & -\mathbf{K} & \ldots & (N-1) \mathbf{K}
\end{array}\right]
$$

where $\mathbf{K}=k \mathbf{I}_{2} \in \mathbb{R}^{2 \times 2}$.

\subsection{Problem Formulation}

Consider a group of $N$ homogenous robots moving in formation, where each agent is defined by

$$
\begin{aligned}
\dot{\mathbf{x}}_{i} & =\mathbf{A} \mathbf{x}_{i}+\mathbf{B} \mathbf{u}_{i}-\mathbf{Z} \sum_{j \in \mathcal{V}_{i}} \gamma_{k, i j}\left(\mathbf{x}_{i}-\mathbf{x}_{j}\right) \\
\mathbf{y}_{i} & =\mathbf{C} \mathbf{x}_{i}
\end{aligned}
$$

Here, $\mathbf{x}_{i}=\left[\mathbf{q}_{i}^{T} \dot{\mathbf{q}}_{i}^{T}\right]^{T} \in \mathbb{R}^{4}$ is the robot state, $\mathbf{u}_{i}=\mathbf{F}_{i} \in$ $\mathbb{R}^{2}$ is the control input, and $\mathbf{y}_{i} \in \mathbb{R}^{2}$ is the output, with

$$
\begin{aligned}
& \mathbf{A}=\left[\begin{array}{llll}
0 & 0 & 1 & 0 \\
0 & 0 & 0 & 1 \\
0 & 0 & 0 & 0 \\
0 & 0 & 0 & 0
\end{array}\right], \mathbf{B}=\left[\begin{array}{cc}
0 & 0 \\
0 & 0 \\
1 / m & 0 \\
0 & 1 / m
\end{array}\right], \\
& \mathbf{Z}=\left[\begin{array}{cccc}
0 & 0 & 0 & 0 \\
0 & 0 & 0 & 0 \\
k / m & 0 & 0 & 0 \\
0 & k / m & 0 & 0
\end{array}\right], \mathbf{C}=\left[\begin{array}{ll}
1 & 0 \\
0 & 1 \\
0 & 0 \\
0 & 0
\end{array}\right]
\end{aligned}
$$

The Laplacian matrix describing the physical interactions between robots as a result of the spring elements is defined as $\mathcal{L}_{k} \in \mathbb{R}^{N \times N}$, where $l_{k, i i}=\sum_{j \neq i} \gamma_{k, i j}$ and $l_{k, i j}=-\gamma_{k, i j}$ for all $i \neq j$. Here, $\gamma_{k, i j}$ is 1 for connected nodes and 0 for unconnected nodes. Accordingly, $\left[\mathcal{L}_{k} \otimes \mathbf{w}_{k}\right]_{i}=\mathbf{Z} \sum_{j \in \mathcal{V}_{i}} \gamma_{k, i j}\left(\mathbf{x}_{i}-\mathbf{x}_{j}\right)$, where $\mathbf{w}_{k_{i}}=\mathbf{Z}_{\mathbf{x}_{i}} \in \mathbb{R}^{4}$ and $\mathbf{w}_{k}=\left[\mathbf{w}_{k_{1}}^{T} \mathbf{w}_{k_{2}}^{T} \ldots \mathbf{w}_{k_{N}}^{T}\right]^{T}$ are the physical interaction signals. For a fully-connected massspring model, $\gamma_{k, i j}=1$ for all $(i, j)$. It can be shown that $\mathcal{L}_{k}$ satisfies Lemma 1 and is a positive, semi-definite matrix.

The multi-agent system can be formulated as the following state-space representation

$$
\begin{aligned}
& \dot{\mathbf{x}}=\left(\mathbf{I}_{N} \otimes \mathbf{A}-\mathcal{L}_{k} \otimes \mathbf{Z}\right) \mathbf{x}+\left(\mathbf{I}_{N} \otimes \mathbf{B}\right) \mathbf{u} \\
& \mathbf{y}=\left(\mathbf{I}_{N} \otimes \mathbf{C}\right) \mathbf{x}
\end{aligned}
$$

where $\mathbf{x}=\left[\begin{array}{lll}\mathbf{x}_{1}^{T} & \mathbf{x}_{2}^{T} \ldots \mathbf{x}_{N}^{T}\end{array}\right]^{T}, \mathbf{u}=\left[\begin{array}{llll}\mathbf{u}_{1}^{T} & \mathbf{u}_{2}^{T} & \ldots & \mathbf{u}_{N}^{T}\end{array}\right]^{T}$, and $\mathbf{y}=\left[\begin{array}{llll}\mathbf{y}_{1}^{T} & \mathbf{y}_{2}^{T} & \ldots & \mathbf{y}_{N}^{T}\end{array}\right]^{T}$.

\section{LQR-Based Optimal Control Design}

LQR is a well-known structured design technique for optimal control of a linear system. This section explores how LQR design method can be used to achieve cooperative consensus control of robot formations.

\subsection{Hierarchical LQR Control Design}

A hierarchical LQR controller is proposed to simultaneously achieve trajectory tracking and formationkeeping, which is presented as a multi-agent consensus problem. If the object being transported is of sufficient stiffness, the physical force interaction produced by the connecting spring elements would act to maintain consensus. However, less rigid objects produce lower interaction, which can lead to significant deformation of geometry. Therefore, hierarchical LQR control introduces an additional information exchange term that ensures Lemma 4 is satisfied, regardless of the material being transported. Fig. 2 describes this hierarchical, decentralized control system. 


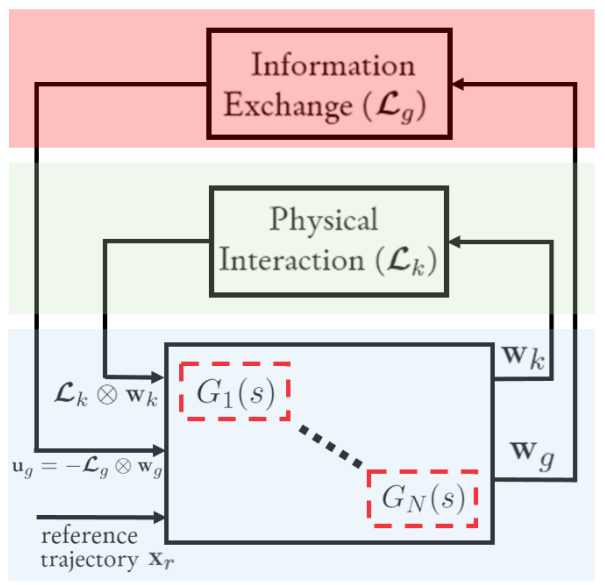

Fig. 2. Block diagram of the hierarchical, decentralized control system.

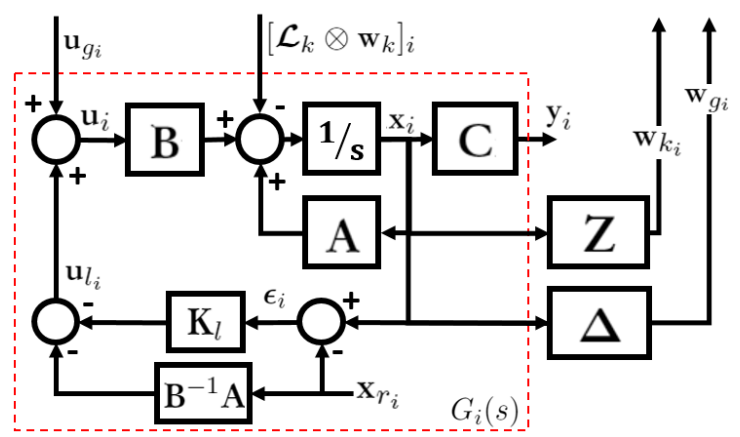

Fig. 3. Block diagram of robot $\mathbf{q}_{i}$. The locally controlled closed-loop system $G_{i}(s)$ is shown in the red box.

Fig. 3 shows the control structure of robot $\mathbf{q}_{i}$, where the proposed control scheme is defined as

$$
\mathbf{u}_{i}=\mathbf{u}_{l_{i}}+\mathbf{u}_{g_{i}}
$$

Here, $\mathbf{u}_{l_{i}}$ is the local control input to achieve trajectory tracking and $\mathbf{u}_{g_{i}}$ is the global control input to establish consensus.

\subsection{Local LQR Controller Design}

To design the local LQR controller for trajectory tracking, all physical force interactions between robots are ignored (i.e. $\mathcal{L}_{k}=\mathbf{0}$ ), and the global control input is assumed be zero (i.e. $\mathbf{u}_{g_{i}}=\mathbf{0}$ ).

Define the local trajectory tracking error as

$$
\boldsymbol{\epsilon}_{i}=\mathbf{x}_{i}-\mathbf{x}_{r_{i}}
$$

where $\mathbf{x}_{r_{i}}$ is the reference trajectory for robot $\mathbf{q}_{i}$.

The local control signal $\mathbf{u}_{l_{i}}$ is designed as

$$
\mathbf{u}_{l_{i}}=-\mathbf{K}_{l} \boldsymbol{\epsilon}_{i}-\mathbf{B}^{-1} \mathbf{A} \mathbf{x}_{r_{i}}
$$

where $\mathbf{K}_{l}$ is the control gain matrix designed using $L Q R$ to minimize the performance index of a quadratic cost function given by $\mathbf{J}_{i}=\int_{0}^{\infty}\left(\boldsymbol{\epsilon}_{i}^{T} \mathbf{Q} \boldsymbol{\epsilon}_{i}+\mathbf{u}_{l_{i}}^{T} \mathbf{R} \mathbf{u}_{l_{i}}\right) d t$. Let
$\mathrm{Q}=\mathbf{Q}^{T} \in \mathbb{R}^{2 \times 2}$ and $\mathbf{R}=\mathbf{R}^{T} \in \mathbb{R}^{2 \times 2}$ be positive definite design matrices; then $\mathbf{K}_{l}$ is obtained by

$$
\mathbf{K}_{l}=\mathbf{R}^{-1} \mathbf{B}^{T} \mathbf{P}
$$

where $\mathbf{P}=\mathbf{P}^{T}>0$ is the solution of the following algebraic Riccati equation

$$
\mathbf{P A}+\mathbf{A}^{T} \mathbf{P}-\mathbf{P B R}^{-1} \mathbf{B}^{T} \mathbf{P}+\mathbf{Q}=0
$$

The local closed-loop system of robot $\mathbf{q}_{i}$ becomes

$$
\begin{aligned}
\dot{\mathbf{x}}_{i} & =\mathbf{A} \mathbf{x}_{i}+\mathbf{B} \mathbf{u}_{i} \\
& =\mathbf{A} \mathbf{x}_{i}-\mathbf{B K} \boldsymbol{\epsilon}_{l}-\mathbf{A} \mathbf{x}_{r_{i}} \\
& =\left(\mathbf{A}-\mathbf{B} \mathbf{K}_{l}\right) \boldsymbol{\epsilon}_{i}
\end{aligned}
$$

Theorem 1. The local control law (Eq. 10) guarantees asymptotic stability of the local trajectory tracking error $\boldsymbol{\epsilon}_{i}$ as $t \rightarrow \infty$.

Proof. Consider the following Lyapunov candidate function

$$
V_{\boldsymbol{\epsilon}_{i}}=\boldsymbol{\epsilon}_{i}^{T} \mathbf{P} \boldsymbol{\epsilon}_{i}
$$

Without loss of generality, it is assumed that the reference trajectory is time-invariant such that $\dot{\mathbf{x}}_{r_{i}}=0$. Taking the derivative and making use of Eq. 13 yields

$$
\begin{aligned}
\dot{V}_{\boldsymbol{\epsilon}_{i}} & =\dot{\boldsymbol{\epsilon}}_{i}^{T} \mathbf{P} \boldsymbol{\epsilon}_{i}+\boldsymbol{\epsilon}_{i}^{T} \mathbf{P} \dot{\boldsymbol{\epsilon}}_{i} \\
& =\boldsymbol{\epsilon}_{i}^{T}\left(\mathbf{A}^{T}-\mathbf{K}_{l}^{T} \mathbf{B}^{T}\right) \mathbf{P} \boldsymbol{\epsilon}_{i}+\boldsymbol{\epsilon}_{i}^{T} \mathbf{P}\left(\mathbf{A}-\mathbf{B} \mathbf{K}_{l}\right) \boldsymbol{\epsilon}_{i} \\
& =\boldsymbol{\epsilon}_{i}^{T}\left(\mathbf{A}^{T} \mathbf{P}-\mathbf{K}_{l}^{T} \mathbf{B}^{T} \mathbf{P}\right) \boldsymbol{\epsilon}_{i}+\boldsymbol{\epsilon}_{i}^{T}\left(\mathbf{P A}-\mathbf{P B K} \mathbf{K}_{l}\right) \boldsymbol{\epsilon}_{i} \\
& =\boldsymbol{\epsilon}_{i}^{T}\left(\mathbf{A}^{T} \mathbf{P}+\mathbf{P A}-\mathbf{P B R} \mathbf{R}^{-1} \mathbf{B}^{T} \mathbf{P}-\mathbf{P B R} \mathbf{R}^{-1} \mathbf{B}^{T} \mathbf{P}\right) \boldsymbol{\epsilon}_{i} \\
& =-\boldsymbol{\epsilon}_{i}^{T}\left(\mathbf{Q}+\mathbf{P B R} \mathbf{R}^{-1}\left(\mathbf{R R}^{-1}\right) \mathbf{B}^{T} \mathbf{P}\right) \boldsymbol{\epsilon}_{i} \\
& =-\boldsymbol{\epsilon}_{i}^{T}\left(\mathbf{Q}+\mathbf{K}_{l}^{T} \mathbf{R} \mathbf{K}_{l}\right) \boldsymbol{\epsilon}_{i}
\end{aligned}
$$

Since $\mathbf{Q}>0$ and $\mathbf{R}>0$, then $\left(\mathbf{Q}+\mathbf{K}_{l}^{T} \mathbf{R} \mathbf{K}_{l}\right)>0$. Therefore $\dot{V}_{\boldsymbol{\epsilon}_{i}}<0$, and the asymptotic stability of the local trajectory tracking error $\epsilon_{i}$ is guaranteed.

\subsection{Global Controller Design}

The global control signal for robot $\mathbf{q}_{i}$ is designed as

$$
\mathbf{u}_{g_{i}}=-\Delta \sum_{j=1}^{N} \gamma_{g, i j}\left(\mathbf{x}_{i}-\mathbf{x}_{j}\right)
$$

where

$$
\boldsymbol{\Delta}=\left[\begin{array}{ll}
\delta & 0 \\
0 & 0
\end{array}\right]
$$

and $\delta \geq 0$ is a consensus gain. Information exchange between robots is indicated by $\gamma_{g, i j}$, which is 1 if a communication channel exists between robots $\mathbf{q}_{i}$ and $\mathbf{q}_{j}$, or 0 otherwise.

This leads to the definition of the global control signal $\mathbf{u}_{g}$ for all nodes as

$$
\mathbf{u}_{g}=-\left(\mathcal{L}_{g} \otimes \boldsymbol{\Delta}\right) \mathbf{x}
$$

where $\mathcal{L}_{g} \in \mathbb{R}^{N \times N}$ is the Laplacian matrix representing information exchange between nodes defined as $l_{g, i i}=$ 
$\sum_{j \neq i} \gamma_{g, i j}$ and $l_{g, i j}=-\gamma_{g, i j}$ for all $i \neq j$. The aggregate signals are defined as $\mathbf{w}_{g_{i}}=\boldsymbol{\Delta} \mathbf{x}_{i} \in \mathbb{R}^{4}$ and $\mathbf{w}_{g}=\left[\mathbf{w}_{g_{1}}^{T} \mathbf{w}_{g_{2}}^{T} \ldots \mathbf{w}_{g_{N}}^{T}\right]^{T}$. A necessary condition on the communication topology of the system is that $\mathcal{L}_{g}$ is connected and satisfies Lemma 1.

The average state of all nodes is given as

$$
\overline{\mathbf{x}}=\frac{1}{N} \sum_{i=1}^{N} \mathbf{x}_{i}
$$

Then the average consensus error of robot $\mathbf{q}_{i}$ is defined as

$$
\mathbf{e}_{i}=\mathbf{x}_{i}-\overline{\mathbf{x}}, \quad i \in\{1, \ldots, N\}
$$

and measures the disagreement of $\mathbf{x}_{i}$ to the average state of all nodes $\overline{\mathbf{x}}$. Let $\mathbf{e}=\left[\mathbf{e}_{1}^{T} \mathbf{e}_{2}^{T} \ldots \mathbf{e}_{N}^{T}\right]^{T} \in \mathbb{R}^{4 N}$. Note that if $\mathbf{e}=\mathbf{0}$ is satisfied, then $\mathbf{x}_{i}=\mathbf{x}_{j}$ holds $\forall i, j \in \mathcal{V}_{i}$, such that Lemma 4 is satisfied and consensus of the system is achieved [38]. This implies that e can quantitatively reflect the degree of disagreement, where a small value of $\|\mathbf{e}\|$ indicates good consensus performance of the system. Note that the average consensus error $\mathbf{e}$ for all nodes is

$$
\mathrm{e}=\mathrm{x}-\overline{\mathrm{x}} \mathbf{1}
$$

Theorem 2. The global control law (Eq. 17) guarantees asymptotic stability of the average consensus error $e$ as $t \rightarrow$ $\infty$.

Proof. Consider an undirected graph described by $\mathcal{L}_{g}$, where each node applies the control protocol given in Eq. 15. It can be shown that $\sum_{i=1}^{N} \mathbf{u}_{g_{i}}=\mathbf{0}$. Thus, $\overline{\mathbf{x}}$ is an invariant quantity, i.e. $\dot{\overline{\mathrm{x}}}=0$, such that $\dot{\mathrm{e}}=\dot{\mathrm{x}}$. Assuming all local control signals $\mathbf{u}_{l_{i}}=\mathbf{0}$, then the global control law specified by Eq. 17 results in

$$
\begin{aligned}
\dot{\mathbf{x}} & =\left(\mathbf{I}_{N} \otimes \mathbf{A}-\mathcal{L}_{k} \otimes \mathbf{Z}\right) \mathbf{x}+\left(\mathbf{I}_{N} \otimes \mathbf{B}\right) \mathbf{u} \\
& =\left(\mathbf{I}_{N} \otimes \mathbf{A}-\mathcal{L}_{k} \otimes \mathbf{Z}-\mathcal{L}_{g} \otimes \mathbf{B} \boldsymbol{\Delta}\right) \mathbf{x} \\
& =\overline{\mathbf{A}} \mathbf{x}
\end{aligned}
$$

Therefore, a necessary and sufficient condition for consensus depends on $\overline{\mathbf{A}}$. If $\left(\mathbf{I}_{N} \otimes \mathbf{A}-\mathcal{L}_{k} \otimes \mathbf{Z}\right)$ is Hurwitz, then formation consensus is achieved for any value of $\delta \geq 0$. Otherwise, if $\delta$ can be chosen such that $\overline{\mathbf{A}}$ becomes Hurwitz, then formation consensus is guaranteed for $\delta \geq \underline{\delta}$, where $\underline{\delta}$ is a positive constant determined by the eigenvalues of the closed-loop system. Note that the eigenvalues of $\overline{\mathbf{A}}$ is related to the rate of convergence to the average consensus state.

\section{Simulation Results and Discussions}

The effectiveness and scalability of hierarchical, decentralized LQR control are evaluated through computer simulations of the cooperative object transport task. The simulation is designed based on omni-

\begin{tabular}{|l|c|}
\hline Material & Stiffness, $k(\mathrm{~N} / \mathrm{m})$ \\
\hline Rubber & 1.45 \\
Steel & 30000 \\
\hline
\end{tabular}

Table 1. Stiffness values of transport object materials.

directional robots, such as employed in many industrial applications. The dynamic model and driving mechanisms of these robots can be found in [14]. Each robot is assumed to have a mass of $40 \mathrm{~kg}$, which is comparable to similar industrial robots used in [4] and acts as the dynamic constraint on the system. Rubber and steel are selected as materials to represent transport objects with low and high rigidity, respectively. The stiffness $k$ of each material is shown in Table 1.

The simulation begins with the aggregation of $N$ robots from random initial positions to the starting location, creating a formation with a predefined geometric shape and size that matches the transport object. Once the transport object is placed on top of the robots, a trajectory is generated for the formation to reach a target location, using artificial potential function and existing knowledge of the environment to avoid known obstacles. In the event that a previously unknown obstacle is detected along the path by the on-board sensors of each robot, additional local collision avoidance protocols would be taken. It is assumed that the unknown obstacle may not be detected by some robots in the formation, due to line-of-sight or range limitations. Two geometric formations are considered to evaluate the proposed method: a line formation consisting of two robots and a quadrilateral formation with four robots.

\subsection{Line Formation with Two Robots}

The first simulation involves two robots, denoted as $\mathrm{q}_{1}$ and $\mathrm{q}_{2}$, that forms a line formation with an equilibrium distance of $r_{12}=1 \mathrm{~m}$. The local controller of each robot is designed using $\mathrm{LQR}$ with $\mathrm{Q}=\mathbf{I}_{4}$ and $\mathbf{R}=\mathbf{I}_{2}$, such that the optimal feedback gain given by Eq. 11 is $\mathbf{K}_{l}=[1,0,9,0 ; 0,1,0,9]$. The initial positions of the

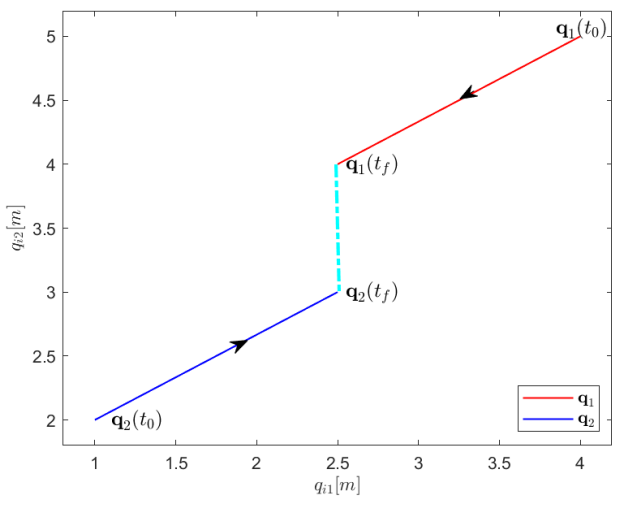

Fig. 4. Aggregation of two robots to the line formation in Cartesian space. 
robots are $\mathbf{q}_{1}\left(t_{0}\right)=[4,5]^{T}$ and $\mathbf{q}_{2}\left(t_{0}\right)=[1,2]^{T}$, respectively. Two unknown, static obstacles are assumed to be located at $[6,5.05 ; 20,1.9]^{T}$ and can only be detected by the nearest robot. Local performance is evaluated by considering the trajectory tracking error of each robot. Formation-keeping is measured by the consensus error between robots.

\subsubsection{Aggregation to Line Formation}

During the aggregation process, the robots $\mathrm{q}_{1}$ and $\mathrm{q}_{2}$ travel from their initial positions to the starting location of the formation, forming a straight line with a relative distance of $1 \mathrm{~m}$. This is shown in Fig. 4 and Fig. 5, where the positions of the robots after aggregation are $\mathbf{q}_{1}\left(t_{f}\right)=[2.5,3]^{T}$, and $\mathbf{q}_{2}\left(t_{f}\right)=[2.5,4]^{T}$. The performance of three consensus gain values $(\delta)$ are compared, with the $L^{2}$-norm of the consensus error $(\|\mathbf{e}\|)$ shown in Fig. 6. It is noted that higher values of $\delta$ result in faster aggregation time. Once the initial formation is established, the group may begin moving towards the target location using either local or hierarchical LQR control.

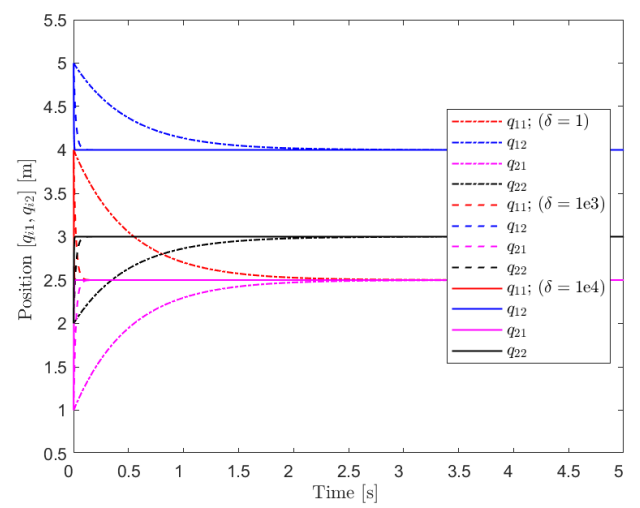

Fig. 5. Aggregation of two robots to the line formation for three different consensus gains $(\delta)$.

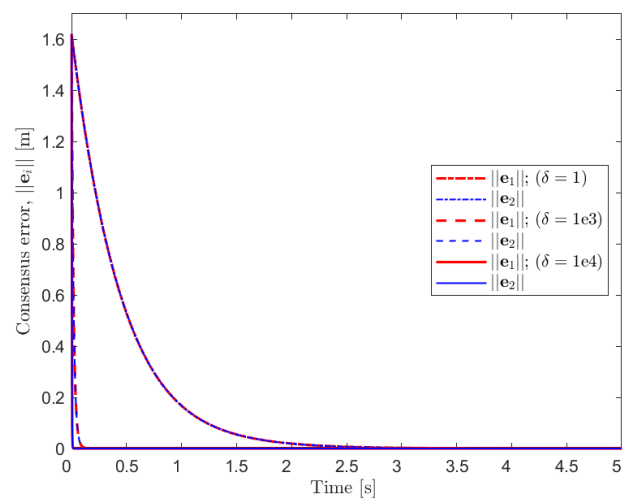

Fig. 6. Consensus error during aggregation to line formation.

\subsubsection{Trajectory Tracking and Formation-Keeping with Line Formation}

Hierarchical LQR control is implemented to simultaneously achieve trajectory tracking and formationkeeping. The trajectory of the robot formation as it moves from the starting location to the target location is shown in Fig. 7 when the consensus gain $\delta=0$. This implies that no information is exchanged between $\mathrm{q}_{1}$ and $\mathrm{q}_{2}$, and the controller is reduced to local LQR control (Eq. 10). Initially, both robots are able to maintain the equilibrium distance of $1 \mathrm{~m}$. However, as $\mathbf{q}_{1}$ approaches the unknown obstacle and begins to take collision avoidance actions, the relative distance between $q_{1}$ and $q_{2}$ is clearly affected, where it is assumed that $\mathbf{q}_{2}$ is unable to detect the obstacle. The robots' velocities are presented in Fig. 8.
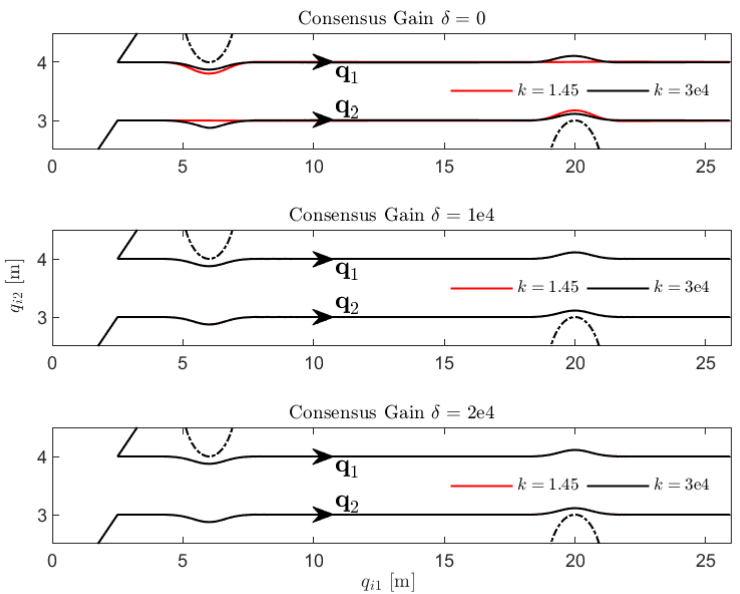

Fig. 7. Trajectory of a 2-robot line formation subjected to two unknown obstacles for different stiffness and consensus gain values.
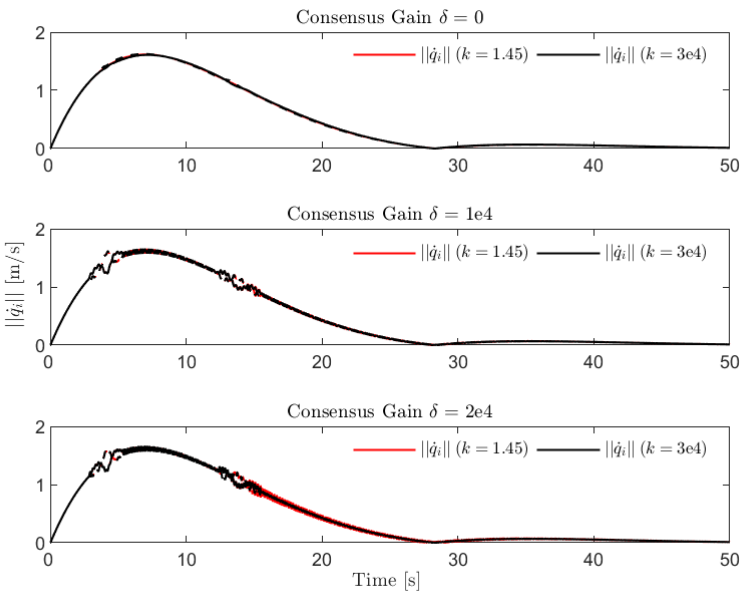

Fig. 8. Robots' velocities of line formation. 


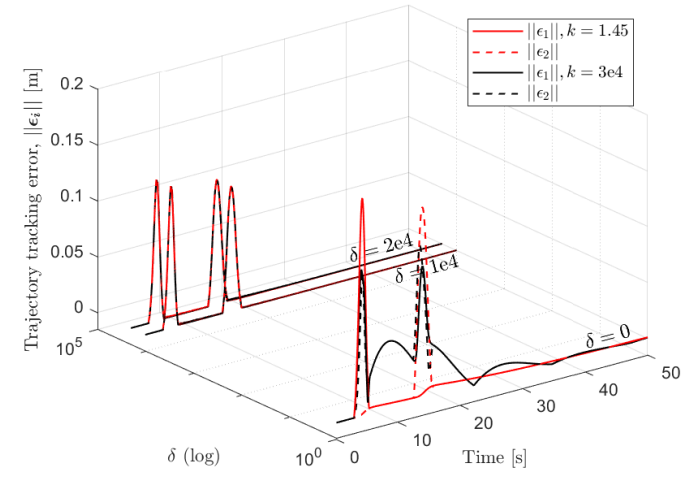

Fig. 9. Trajectory tracking error of a line formation.

\begin{tabular}{|l|c|c|}
\hline Material & $k=1.45$ & $k=3 \mathrm{e} 4$ \\
\hline$\left\|\boldsymbol{\epsilon}_{1}\right\|[\%] ; \delta=0$ & 19.4 & 13 \\
$\left\|\boldsymbol{\epsilon}_{2}\right\|[\%] ; \delta=0$ & 17.1 & 13.8 \\
\hline$\left\|\boldsymbol{\epsilon}_{1}\right\|[\%] ; \delta=1 \mathrm{e} 4$ & 12.5 & 12.6 \\
$\left\|\boldsymbol{\epsilon}_{2}\right\|[\%] ; \delta=1 \mathrm{e} 4$ & 12.8 & 12.7 \\
\hline$\left\|\boldsymbol{\epsilon}_{1}\right\|[\%] ; \delta=2 \mathrm{e} 4$ & 12.6 & 12.6 \\
$\left\|\boldsymbol{\epsilon}_{2}\right\|[\%] ; \delta=2 \mathrm{e} 4$ & 12.7 & 12.7 \\
\hline
\end{tabular}

Table 2. Trajectory tracking error of a line formation.

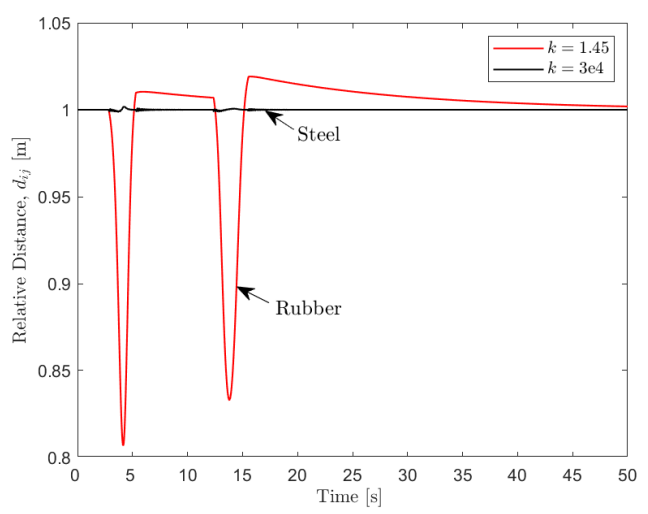

Fig. 10. Relative distance $d_{12}$ between $\mathbf{q}_{1}$ and $\mathbf{q}_{2}$ when $\delta=0$. The equilibrium distance is $1 \mathrm{~m}$.

The trajectory tracking error of the local LQR controller is shown in Fig. 9, with the consensus gain $\delta=0$. The trajectory tracking error is considered using $L^{2}$ norm analysis. The solid and dashed lines for each stiffness value represent $\mathbf{q}_{1}$ and $\mathbf{q}_{2}$, respectively. The tracking error $(\|\epsilon\|)$ is summarized in Table 2 . The degree to which each robot deviates from the reference trajectory depends on the rigidity of the transport object. For an elastic material, such as rubber, $\mathbf{q}_{2}$ remains relatively unaffected by any actions taken by $\mathbf{q}_{1}$. This causes a loss of formation consensus between the two robots, as can be seen by the relative distance $d_{12}$ in Fig. 10. For a rigid object, such as steel, a strong physical interaction force exists between the two robots, allowing the formation to be maintained. This is further examined in Fig. 11 and Table 3 by considering the consensus error between

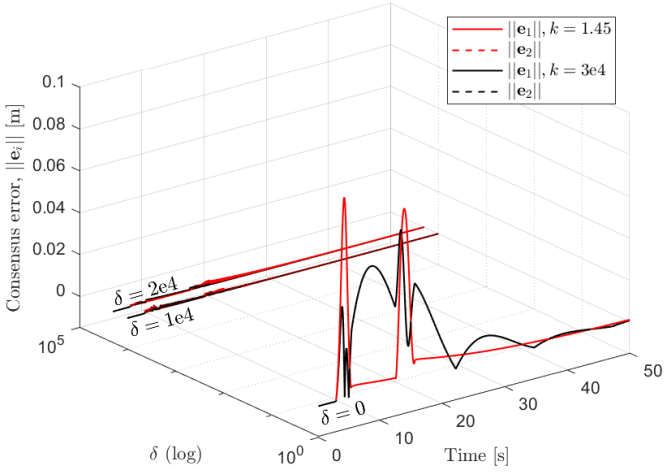

Fig. 11. Consensus error of a line formation. Note that $\left\|\mathbf{e}_{1}\right\|$ and $\left\|\mathbf{e}_{2}\right\|$ are overlapped.

\begin{tabular}{|l|c|c|c|c|}
\hline & \multicolumn{2}{|c|}{$\|\mathbf{e}\|[\%]$} & \multicolumn{2}{c|}{ Control |u| [N] } \\
\hline Material & $k=1.45$ & $k=3 \mathrm{e} 4$ & $k=1.45$ & $k=3 \mathrm{e} 4$ \\
\hline$\delta=0$ & 9.6 & 7.3 & 22.5 & 22.5 \\
\hline$\delta=1 \mathrm{e} 4$ & 0.27 & 0.22 & 45 & 51.5 \\
\hline$\delta=2 \mathrm{e} 4$ & 0.26 & 0.17 & 95 & 65 \\
\hline
\end{tabular}

Table 3. Consensus error and control input of a line formation.

robots. The consensus error is considered using $L^{2}$ norm analysis. When only the local LQR controller is implemented $(\delta=0)$, high stiffness is required to ensure that the formation has minimal deviation.

When the consensus gain has a non-zero value, information exchange between $q_{1}$ and $q_{2}$ is used to help maintain formation through hierarchical LQR control. The trajectory tracking errors for three consensus gain values are shown in Fig. 9 and Table 2, where the tradeoff between local performance (trajectory tracking) and consensus (formation-keeping) is observed. To further study the effect of the consensus gain, the results for the lowest stiffness material $(1.45 \mathrm{~N} / \mathrm{m})$ is considered, as the reduced physical interaction force has been shown to cause large consensus error. Fig. 11 shows the consensus error e between $\mathbf{q}_{1}$ and $\mathbf{q}_{2}$. Note that $\delta=0$ implies a local LQR controller. From Table 3 , it is seen that higher values of $\delta$ allow the formation to maintain the equilibrium distance and achieve consensus, regardless of the stiffness of the transport object. The performance gains are most notable for materials with lower stiffness. It is concluded that the consensus gain $\delta$ acts as a virtual spring that enables the formation to be maintained. However, while higher consensus gains provide better performance, this may result in a significant cost of control effort. For example, $\delta=2 \mathrm{e} 4$ generates marginal consensus improvements over $\delta=1 \mathrm{e} 4$, but required noticeably higher control inputs. Therefore, the consensus gain should be chosen with care in order to reduce the likelihood of actuator saturation. 


\subsection{Quadrilateral Formation with Four Robots}

The scalability of the proposed controller is evaluated by considering a quadrilateral formation with four robots, denoted as $\mathbf{q}_{1}, \mathbf{q}_{2}, \mathbf{q}_{3}$, and $\mathbf{q}_{4}$. The initial positions of the robots are $\mathbf{q}_{1}\left(t_{0}\right)=[0.8,5.1]^{T}$, $\mathbf{q}_{2}\left(t_{0}\right)=[0.25,2.45]^{T}, \mathbf{q}_{3}\left(t_{0}\right)=[2.6,2]^{T}$, and $\mathbf{q}_{4}\left(t_{0}\right)=$ $[3.1,4.5]^{T}$, respectively. Two unknown, static obstacles are assumed to be located at $[6,5.05 ; 20,1.9]^{T}$ and can only be detected by the nearest robot. The local LQR controller is the same as for the line formation.

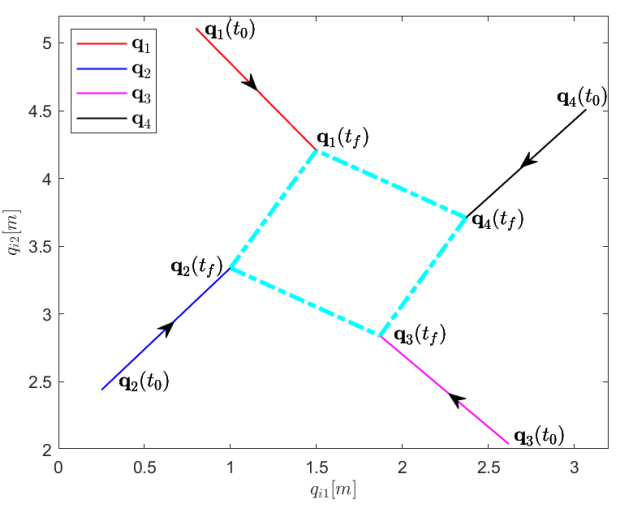

Fig. 12. Aggregation of four robots to the quadrilateral formation in Cartesian space.

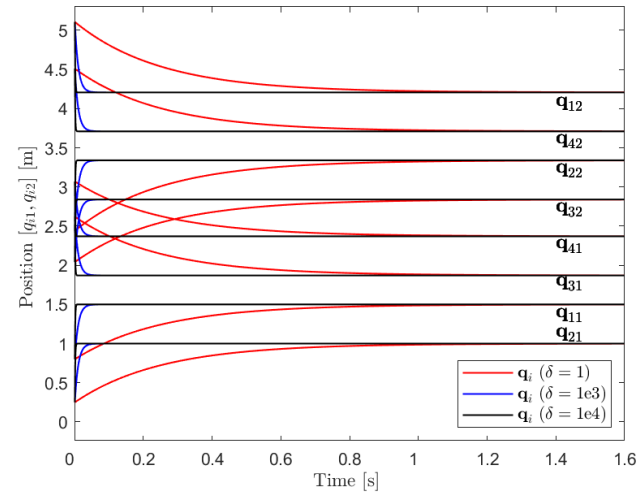

Fig. 13. Aggregation of four robots to the quadrilateral formation for three different consensus gains $(\delta)$.

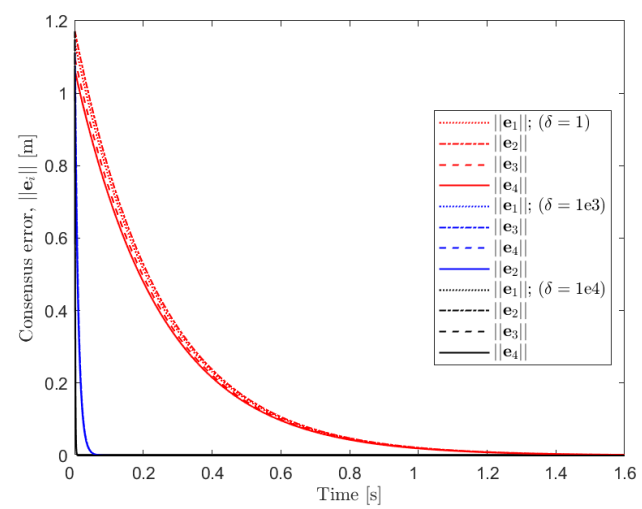

Fig. 14. Consensus error during aggregation to quadrilateral formation.

\subsubsection{Aggregation to Quadrilateral Formation}

In the aggregation phase, the robots travel from their initial positions to the starting location of the formation, forming a quadrilateral geometry with a relative side distance of $1 \mathrm{~m}$. The trajectory of the robots during aggregation and the consensus error for three consensus gain values $(\delta)$ are shown in Fig. 12 to Fig. 14. Again, it is noted that higher values of $\delta$ result in faster aggregation time. Once the aggregation process is completed, the group may start moving towards the target as a quadrilateral formation using either local or hierarchical LQR control.

\subsubsection{Trajectory Tracking and Formation-Keeping with Quadrilateral Formation}

The proposed hierarchical decentralized LQR controller is evaluated by considering the simultaneous trajectory tracking and formation-keeping performance as
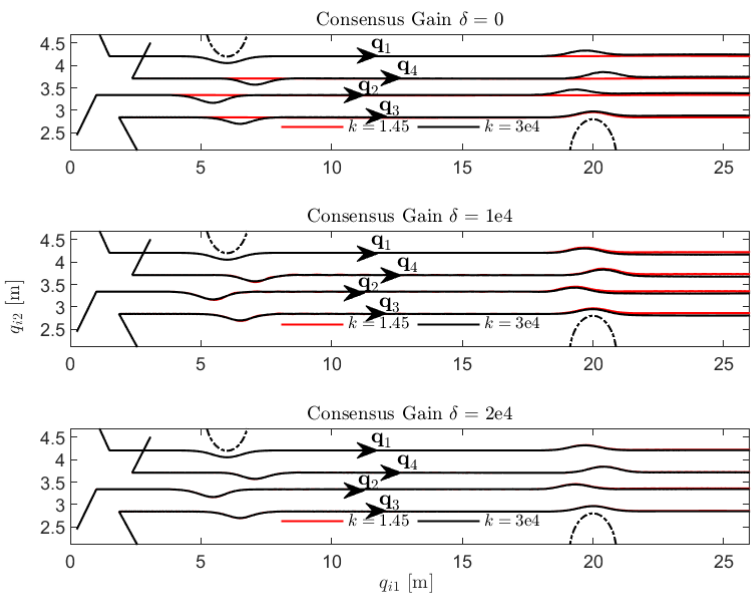

Fig. 15. Trajectory of a 4-robot quadrilateral formation subjected to two unknown obstacles for different stiffness and consensus gain values.
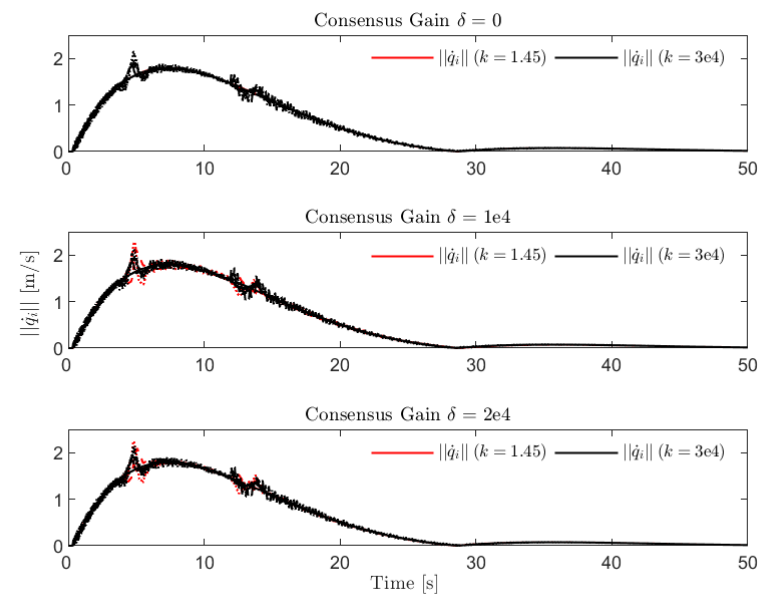

Fig. 16. Robots' velocities of quadrilateral formation. 


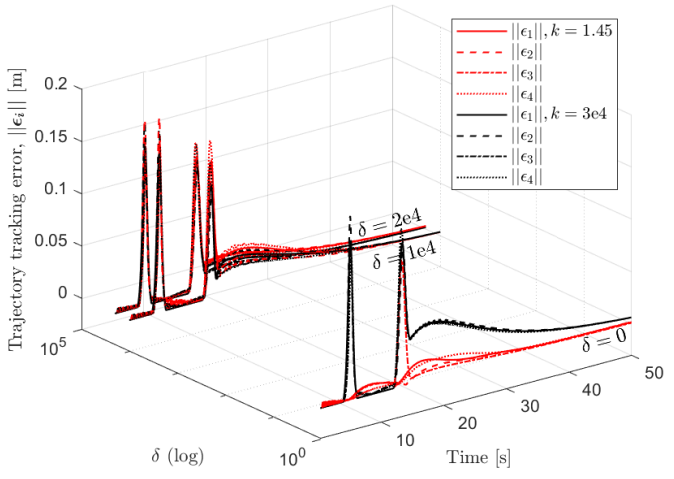

Fig. 17. Trajectory tracking error of a quadrilateral formation.

\begin{tabular}{|l|c|c|}
\hline Material & $k=1.45$ & $k=3 \mathrm{e} 4$ \\
\hline$\|\boldsymbol{\epsilon}\|[\%] ; \delta=0$ & 15 & 17.6 \\
\hline$\|\boldsymbol{\epsilon}\|[\%] ; \delta=1 \mathrm{e} 4$ & 18.6 & 17.5 \\
\hline$\|\boldsymbol{\epsilon}\|[\%] ; \delta=2 \mathrm{e} 4$ & 17.7 & 17.4 \\
\hline
\end{tabular}

Table 4. Trajectory tracking error of a quadrilateral formation.

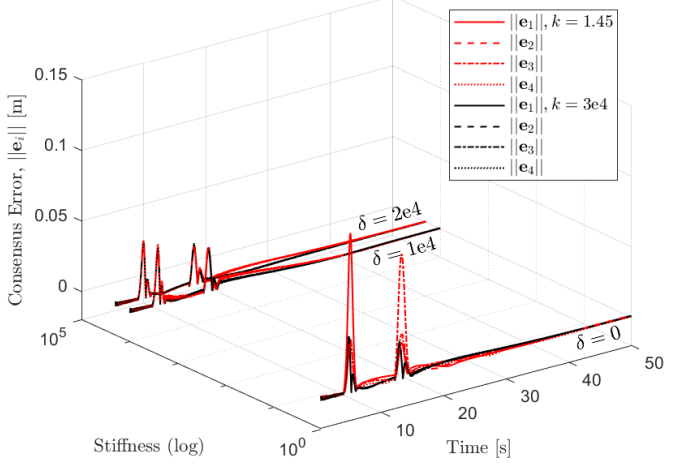

Fig. 18. Consensus error of a quadrilateral formation.

\begin{tabular}{|l|c|c|c|c|}
\hline & \multicolumn{2}{|c|}{$\| \mathbf{e}||[\%]$} & \multicolumn{2}{c|}{ Control $|\mathbf{u}|[\mathrm{N}]$} \\
\hline Material & $k=1.45$ & $k=3 \mathrm{e} 4$ & $k=1.45$ & $k=3 \mathrm{e} 4$ \\
\hline$\delta=0$ & 11.3 & 4.1 & 25 & 26 \\
\hline$\delta=1 \mathrm{e} 4$ & 4.3 & 4.1 & 142 & 111 \\
\hline$\delta=2 \mathrm{e} 4$ & 4.1 & 4 & 198 & 199 \\
\hline
\end{tabular}

Table 5. Consensus error and control input of a quadrilateral formation.

the robots move towards the target location. The trajectory of the robot formation as it moves from the starting location to the target location is shown in Fig.15. The robots' velocities are presented in Fig. 16. The trajectory tracking error of the local LQR controller with the consensus gain $\delta=0$ is shown in Fig. 17 for two different transport materials and summarized in Table 4. The corresponding consensus error is shown in Fig. 18 and
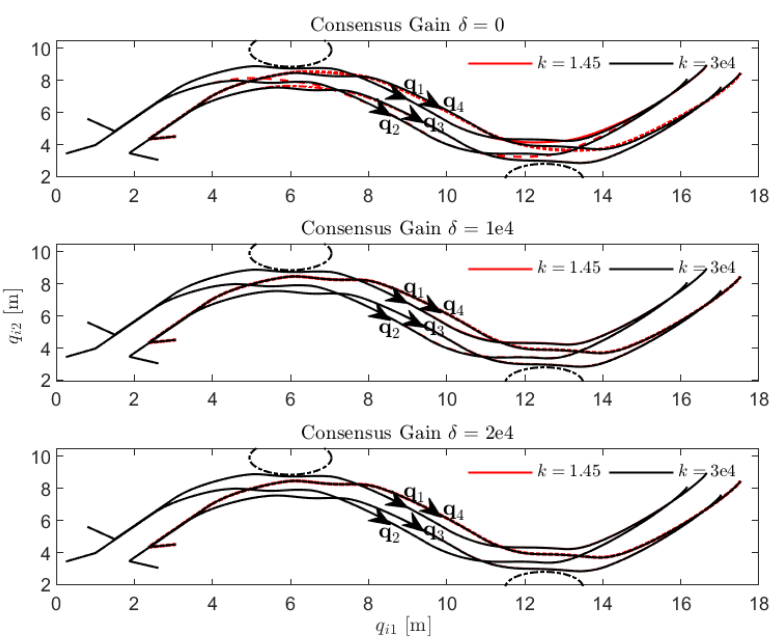

Fig. 19. A 4-robot quadrilateral formation following an s-curve trajectory and subjected to two unknown obstacles.

Table 5. The results indicate that formation is maintained only for high-stiffness materials, such as steel, with strong physical interaction forces between robots.

The trajectory tracking and consensus errors when applying the hierarchical decentralized LQR controller with the consensus gains $\delta=0,1 \mathrm{e} 4$, and 2e 4 are shown in Fig. 17 and Fig. 18, respectively. The errors are summarized and compared to the local LQR controller in Table 4 and Table 5 . It is shown that the consensus gain $\delta$ enables all four robots to maintain a quadrilateral formation, regardless of the stiffness of the transport materials. Again, it is noted that higher consensus gains may provide marginal improvements, but at a significant cost in control efforts. The proposed algorithm was also tested for different trajectories, such as an s-curve, as shown in Fig. 19.

\subsection{Scalability}

The proposed method is scalable to formations involving more robots. To illustrate this, additional analysis was conducted for an octagonal formation with 8 robots. This is compared to the previously considered 2- and 4-robot formations in Fig. 20. It can be seen that the number of physical interactions between robots increased significantly. The trajectory of the 8-robot formation subjected to two unknown obstacles and different stiffness values is shown in Fig. 21. The consensus performance is given in Table 6 . It is noted that the consensus gain $\delta$ is necessary to maintain the formation at low stiffness. However, only marginal improvements are observed for high stiffness materials, while requiring a large amount of control effort. It is concluded that the consensus gain should be kept small for formations involving more robots and rigid objects. 


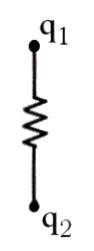

(a)

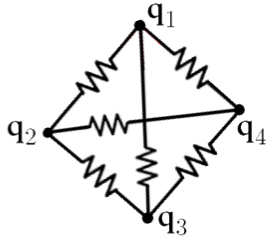

(b)

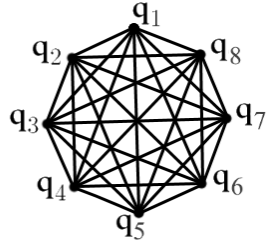

(c)
Fig. 20. (a) 2-robot (line), (b) 4-robot (quadrilateral), and (c) 8-robot (octagonal) formations.
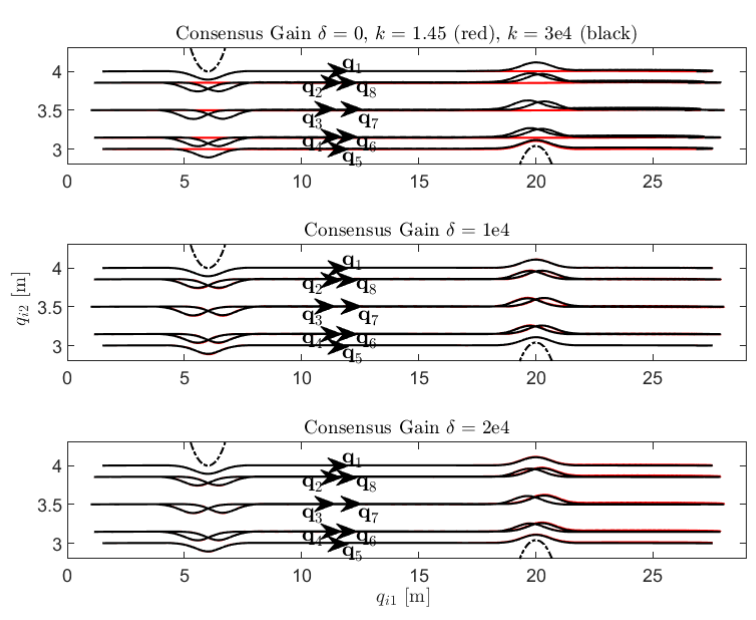

Fig. 21. Trajectory of an 8-robot octagonal formation subjected to two unknown obstacles for different stiffness and consensus gain values.

\begin{tabular}{|l|c|c|c|c|}
\hline & \multicolumn{2}{|c|}{$\|\mathbf{e}\|[\%]$} & \multicolumn{2}{c|}{ Control $|\mathbf{u}|[\mathrm{N}]$} \\
\hline Material & $k=1.45$ & $k=3 \mathrm{e} 4$ & $k=1.45$ & $k=3 \mathrm{e} 4$ \\
\hline$\delta=0$ & 9.3 & 2.3 & 25 & 70.4 \\
\hline$\delta=1 \mathrm{e} 4$ & 2.4 & 2 & 58 & 877 \\
\hline$\delta=2 \mathrm{e} 4$ & 2.3 & 2 & 69.5 & 1730 \\
\hline
\end{tabular}

Table 6. Consensus error and control input of an octagonal formation.

\subsection{Communication Topology}

For the quadrilateral formation with four robots, the proposed controller was implemented assuming a fullyconnected topology that required 12 communication channels. Information exchange between robots guaranteed formation-keeping, regardless of the transport material stiffness. However, a fully-connected, undirected graph results in a relatively high communication cost that increases exponentially with the number of nodes [39]. This implies that there is a trade-off between communication bandwidth and consensus performance. To explore the effect of communication topology on the hierarchical decentralized LQR controller, four different topologies are considered, as shown in Fig. 22. Note that one bidirectional edge requires two communication

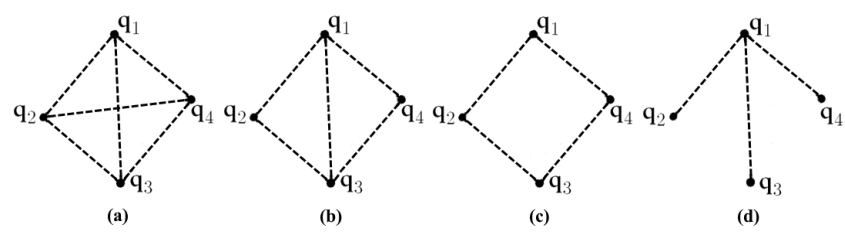

Fig. 22. (a) Communication topologies with 12 channels (fully-connected), (b) 10 channels, (c) 8 channels (cyclic), (d) and 6 channels.

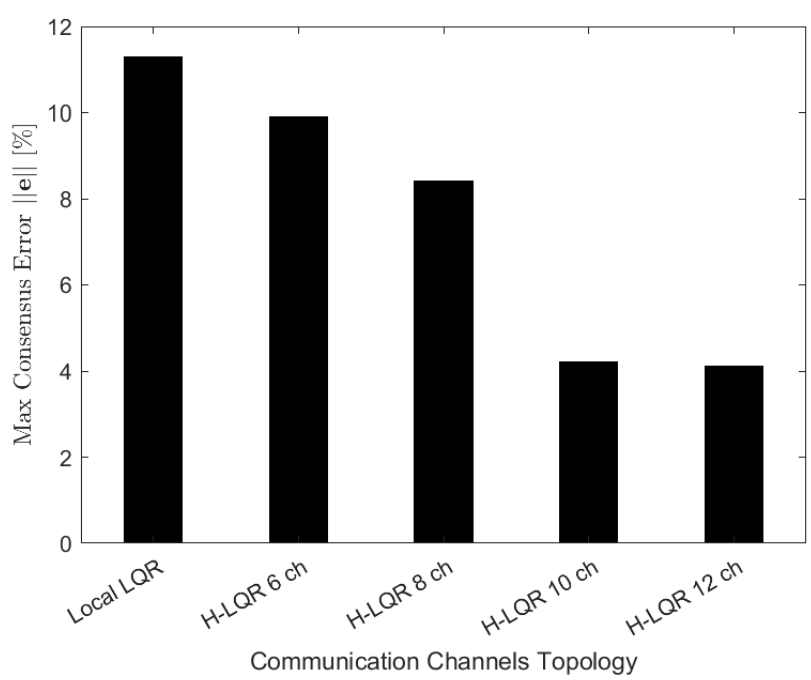

Fig. 23. Comparison of maximum consensus error $\|\mathbf{e}\|$ for low stiffness materials between local LQR and four topologies with gain $\delta=2 \mathrm{e} 4$.

channels. The performance of each topology is evaluated in terms of the maximum consensus error.

Fig. 23 shows the maximum consensus error for each topology. For comparison purposes, the result of a local LQR controller with no information exchange is also included. The hierarchical decentralized LQR controller was implemented with a consensus gain of $\delta=2 \mathrm{e} 4$ for all topologies. It is seen that the 10 channel topology achieves the same performance as a fully-connected topology with 12 channels. The 10channel topology provides acceptable trade-off between communication cost and consensus performance.

\section{Conclusions}

A new method for decentralized formation control with physical interactions between cooperative mobile robots is presented. The formation is maintained when an unknown obstacle is detected and local collision avoidance actions are taken. The strategy uses hierarchical LQR control, with virtual springs implemented through information exchange between robots. This is suitable for the transport of oversized objects in industrial applications, where formation geometry must be kept to avoid damaging the object. The formation is scalable and may form any predefined shape, 
such as line or quadrilateral formations. Stability analysis guarantees local trajectory tracking performance and collision avoidance, while maintaining formation consensus between all robots, regardless of the rigidity of the transport material. Lastly, the effect of communication topology on bandwidth requirements and consensus is presented. Recommended further studies include dynamic changes in communication topology due to delay or channel failure.

\section{Acknowledgement}

This research is financially supported by Sirindhorn International Institute of Technology (SIIT) and Thammasat University (TU).

\section{References}

[1] N. V. Kumar and C. S. Kumar, "Development of collision free path planning algorithm for warehouse mobile robot," Procedia Computer Science, vol. 133, pp. 456-463, 2018.

[2] E. Minca, A. Filipescu, and A. Voda, "Modelling and control of an assembly/disassembly mechatronics line served by mobile robot with manipulator," Control Engineering Practice, vol. 31, pp. 50-62, 2014.

[3] R. Herguedas, G. López-Nicolás, R. Aragüés, and C. Saguiés, "Survey on multi-robot manipulation of deformable objects," IEEE International Conference on Emerging Technologies and Factory Automation, ETFA, vol. 2019-Sept, pp. 977-984, 2019.

[4] K. Piemngam, I. Nilkhamhang, and P. Bunnun, "A virtual spring damper method for formation control of the multi omni-directional robots in cooperative transportation," in 2019 11th International Conference on Information Technology and Electrical Engineering (ICITEE), 2019, pp. 1-6.

[5] F. Ohashi, K. Kaminishi, J. D. F. Heredia, H. Kato, T. Ogata, T. Hara, and J. Ota, "Realization of heavy object transportation by mobile robots using handcarts and outrigger," ROBOMECH Journal, vol. 3, no. 1, Nov. 2016.

[6] N. Yonezawa, K. Kashiwazaki, K. Kosuge, Y. Hirata, Y. Sugahara, M. Endo, T. Kanbayashi, K. Suzuki, K. Murakami, and K. Nakamura, "Car transportation system grasping two drive wheels," in 2012 IEEE International Conference on Robotics and Automation, 2012, pp. 4086-4091.

[7] T. Sugar and V. Kumar, "Decentralized control of cooperating mobile manipulators," in Proceedings. 1998 IEEE International Conference on Robotics and Automation (Cat. No.98CH36146), vol. 4, 1998, pp. 2916-2921.
[8] E. Tuci, M. H. M. Alkilabi, and O. Akanyeti, "Cooperative object transport in multi-robot systems: A review of the state-of-the-art," Frontiers in Robotics and AI, vol. 5, p. 59, 2018.

[9] L. Zhang, Y. Sun, A. Barth, and O. Ma, "Decentralized control of multi-robot system in cooperative object transportation using deep reinforcement learning," IEEE Access, vol. 8, pp. 184 109$184119,2020$.

[10] M. H. M. Alkilabi, A. Narayan, and E. Tuci, "Cooperative object transport with a swarm of e-puck robots: robustness and scalability of evolved collective strategies," Swarm Intelligence, vol. 11, no. 3, pp. 185-209, 2017.

[11] W. Wan, B. Shi, Z. Wang, and R. Fukui, "Multirobot object transport via robust caging," IEEE Transactions on Systems, Man, and Cybernetics: Systems, vol. 50, no. 1, pp. 270-280, 2020.

[12] B. Hichri, L. Adouane, J.-C. Fauroux, Y. Mezouar, and I. Doroftei, "Cooperative mobile robot control architecture for lifting and transportation of any shape payload," in Distributed Autonomous Robotic Systems. Tokyo: Springer Japan, 2016, pp. 177-191.

[13] J. Alonso-Mora, R. Knepper, R. Siegwart, and D. Rus, "Local motion planning for collaborative multi-robot manipulation of deformable objects," in 2015 IEEE international conference on robotics and automation (ICRA). IEEE, 2015, pp. 54955502.

[14] F. Huzaefa and Y.-C. Liu, "Centralized control architecture for cooperative object transportation using multiple omnidirectional agvs," in 2019 IEEE/RSJ International Conference on Intelligent Robots and Systems (IROS). IEEE, 2019, pp. 6526-6532.

[15] A. Rauniyar, H. C. Upreti, A. Mishra, and P. Sethuramalingam, "Mewbots: Mecanumwheeled robots for collaborative manipulation in an obstacle-clustered environment without communication," Journal of Intelligent \& Robotic Systems, vol. 102, no. 1, pp. 1-18, 2021.

[16] T. Machado, T. Malheiro, S. Monteiro, W. Erlhagen, and E. Bicho, "Multi-constrained joint transportation tasks by teams of autonomous mobile robots using a dynamical systems approach," in 2016 IEEE International Conference on Robotics and Automation (ICRA), 2016, pp. 3111-3117.

[17] Z. Wang, G. Yang, X. Su, and M. Schwager, OuijaBots: Omnidirectional Robots for Cooperative Object Transport with Rotation Control Using No Communication. Cham: Springer International Publishing, 2018, pp. 117-131. 
[18] J.-Y. Jhang, C.-J. Lin, and K.-Y. Young, "Cooperative carrying control for multi-evolutionary mobile robots in unknown environments," Electronics, vol. 8, no. 3, 2019.

[19] H. Wicaksono and I. Nilkhamhang, "Glocal controller-based formation control strategy for flexible material handling," in 2017 56th Annual Conference of the Society of Instrument and Control Engineers of Japan (SICE), 2017, pp. 787-792.

[20] M. M. Gulzar, S. T. H. Rizvi, M. Y. Javed, U. Munir, and H. Asif, "Multi-agent cooperative control consensus: A comparative review," Electronics, vol. 7, no. 2, 2018.

[21] K.-K. Oh, M.-C. Park, and H.-S. Ahn, "A survey of multi-agent formation control," Automatica, vol. 53, pp. 424-440, Mar 2015.

[22] A. D. Dang, H. M. La, T. Nguyen, and J. Horn, "Formation control for autonomous robots with collision and obstacle avoidance using a rotational and repulsive force-based approach," International Journal of Advanced Robotic Systems, vol. 16, no. 3, pp. 1-6, 2019.

[23] H. Rezaee and F. Abdollahi, "A decentralized cooperative control scheme with obstacle avoidance for a team of mobile robots," IEEE Transactions on Industrial Electronics, vol. 61, pp. 347-354, 2014.

[24] G. Wen, C. L. Chen, and Y. J. Liu, "Formation Control With Obstacle Avoidance for a Class of Stochastic Multiagent Systems," IEEE Trans. on IndustrialElect., vol. 65, no. 7, pp. 5847-5855, 2018.

[25] B. Young, R. Beard, and J. Kelsey, "A control scheme for improving multi-vehicle formation maneuvers," in Proceedings of the 2001 American Control Conference. (Cat. No.01CH37148), vol. 2, 2001, pp. 704-709.

[26] G.-P. Liu and S. Zhang, "A survey on formation control of small satellites," Proceedings of the IEEE, vol. 106, no. 3, pp. 440-457, Mar. 2018.

[27] A. Alfaro and A. Morán, "Leader-follower formation control of nonholonomic mobile robots," in 2020 IEEE ANDESCON, 2020, pp. 1-6.

[28] C. C. Loh and A. Traechtler, "Cooperative transportation of a load using nonholonomic mobile robots," Procedia Engineering, vol. 41, pp. 860866, 2012.

[29] M. Udomkun and P. Tangamchit, "Cooperative Overhead transportation of a box by decentral- ized mobile robots," 2008 IEEE International Conference on Robotics, Automation and Mechatronics, RAM 2008, pp. 1161-1166, 2008.

[30] W. Ren and R. Beard, "Virtual structure based spacecraft formation control with formation feedback," in AIAA Guidance, Navigation, and Control Conference and Exbibit. American Institute of Aeronautics and Astronautics, Jun. 2002.

[31] M. T. Thai and D. . Du, "Connected dominating sets in disk graphs with bidirectional links," IEEE Communications Letters, vol. 10, no. 3, pp. 138140, 2006.

[32] X. Li, Y. Tang, and H. R. Karimi, "Consensus of multi-agent systems via fully distributed eventtriggered control," Automatica, vol. 116, pp. 1-9, 2020.

[33] R. Olfati Saber and R. M. Murray, "Consensus Protocols for Networks of Dynamic Agents," Proceedings of the American Control Conference, vol. 2, pp. 951-956, 2003.

[34] J. Bai, G. Wen, A. Rahmani, and Y. Yu, "Distributed consensus tracking for the fractional-order multi-agent systems based on the sliding mode control method," Neurocomputing, vol. 235, no. December 2016, pp. 210-216, 2017.

[35] A. Tiwari and S. Devasia, "Rapid transitions with robust accelerated delayed self reinforcement for consensus-based multi agent networks," ArXiv, vol. abs/2006.16295, 2020.

[36] C. X. Shi, G. H. Yang, and X. J. Li, "Robust adaptive backstepping control for hierarchical multiagent systems with signed weights and system uncertainties," IET Control Theory and Applications, vol. 11, no. 16, pp. 2743-2752, 2017.

[37] C. Li, L. Chen, Y. Guo, and G. Ma, "Formationcontainment control for networked EulerLagrange systems with input saturation," Nonlinear Dynamics, vol. 91, pp. 1307-1320, 2018.

[38] Y. Liu and Y. Jia, "H $\infty$ consensus control for multi-agent systems with linear coupling dynamics and communication delays," International Journal of Systems Science, vol. 43, no. 1, pp. 50-62, 2012.

[39] R. Olfati-Saber and R. M. Murray, "Consensus problems in networks of agents with switching topology and time-delays," pp. 1520-1533, 2004. 


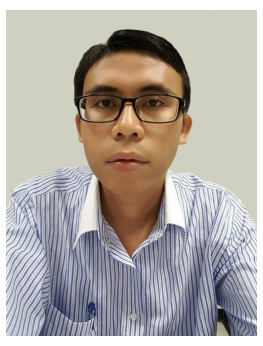

Hendi Wicaksono Agung earned the B.Eng. degree in Electrical Engineering from University of Surabaya at Indonesia, in 2004, and his M.Eng. degree in Electrical Engineering from the Sepuluh Nopember Institute of Technology (ITS) at Indonesia in 2009. He is currently pursuing his Ph.D. degree in Engineering and Technology at Sirindhorn International Institute of Technology, Thammasat University, under the supervision of Dr. Itthisek Nilkhamhang. His research interests include robotics, especially multi-robots, control system, and mechatronics.

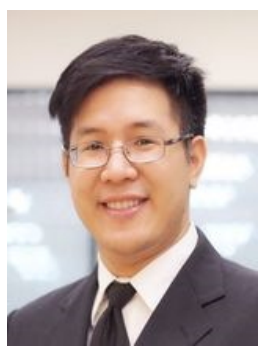

Itthisek Nilkhamhang received his B.Eng. in Electrical Engineering from Sirindhorn International Institute of Technology, Thammasat University, in 2002, and his M.Eng. and Ph.D. in Integrated Design Engineering from Keio University in 2005 and 2008, respectively. He joined Sirindhorn International Institute of Technology, Thammasat University, as a full-time faculty member in 2008. He is currently an assistant professor in the School of Information, Computers, and Communications Technology, as a member of the Electrical Engineering Program. His research interest include iterative tuning and learning algorithms, robust and adaptive control systems, system identification, robotics, mechatronics, instrumentation and automation. 